\title{
O processo de cuidado a pessoa com estomia intestinal
}

\author{
The care process for the person with intestinal ostomy
}

\author{
Bianca dos Santos Blan, Marina Soares Mota, Giovana Calcagno Gomes, Prisciane \\ Cardoso Silva, Juliane Portella Ribeiro
}

Como citar este artigo:

BLAN, BIANCA S.; MOTA, MARINA

S.; COMES, GIOVANA C.; SILVA,

PRISCIANE C.; RIBEIRO, JULIANE

P.; $\bigcirc$ processo de cuidado à

pessoa com estomia intestinal.

Revista Saúde (Sta. Maria). 2020;

46 (2).

\section{Autor correspondente:}

Nome: Bianca dos Santos Blan

E-mail: biancablanenf@gmail.com

Telefone: (53) 991030707

Formação Profissional: Enfermeira

formada pela Universidade

Federal do Rio Grande (FURG);

Residente do Programa

de Residência Integrada

Multiprofissional Hospitalar com

ênfase na Atenção à Saúde

Cardiometabólica do adulto

(RIMHAS) - FURG, Rio Grande, RS,

Brasil.

Filiação Institucional: Universidade Federal do Rio Grande

Endereço para correspondência:

Rua: Acre n²84

Bairro: Frederico Ernesto Buchholz

Cidade: Rio Grande

Estado: Rio Grande do Sul

CEP: $96212-080$

Data de Submissão:

1 1/06/2020

Data de aceite:

21/09/2020

Conflito de Interesse: Não há conflito de interesse

\section{(cc) $\mathrm{BY}-\mathrm{NC}-\mathrm{ND}$}

\section{RESUMO}

Objetivo: Conhecer o processo de cuidado à pessoa com estomia intestinal realizado por profissionais de enfermagem de uma Unidade de Clínica Cirúrgica. Método: Trata-se de uma pesquisa qualitativa, descritiva e exploratória realizada no primeiro semestre de 2019, na Unidade de Clínica Cirúrgica de um Hospital Universitário no extremo sul do Brasil. Participaram do estudo 20 profissionais de enfermagem. A coleta dos dados se deu por meio de entrevistas semiestruturadas individuais e únicas que posteriormente foram submetidas à Análise de Conteúdo. Resultados: Verificou-se a disparidade dos conhecimentos técnico-científicos e práticos dos profissionais de enfermagem. Houve fragilidades na realização de orientações e no preparo pré-operatório envolvido no processo de estomização, além do desconhecimento dos processos fisiológicos que se alteram com a cirurgia. No entanto, houve profissionais que se destacaram pelo domínio técnico-científico e pelo cuidado diferenciado à pessoa com estomia intestinal, buscando atender suas especificidades e a realização de orientações com base no nível de entendimento da pessoa sob seu cuidado e sua família. Considerações Finais: Observa-se a fragilidade no cuidado prestado à pessoa com estomia e sua família, entretanto, a educação continuada juntamente com a implementação da Sistematização da Assistência de Enfermagem nas Unidades de Clínica Cirúrgica, são capazes de gerar conhecimentos a partir da realidade dos profissionais e auxiliar esses a construir um cuidado especializado, repercutindo na redução de complicações imediatas ou tardias, melhorando a vivência da transição da pessoa com estomia intestinal para 0 autocuidado.

PALAVRAS-CHAVE: Estomia; Enfermagem; Cuidados de Enfermagem; Autocuidado; Enfermagem perioperatória.

\section{ABSTRACT}

Objective: To learn about the care process for people with intestinal ostomy performed by nursing professionals in a Surgical Clinic Unit. Method: This is a qualitative, descriptive and exploratory research carried out in the first semester of 2019, at the Surgical Clinic Unit of a University Hospital in the extreme south of Brazil. Twenty nursing professionals participated in the study. Data collection took place through individual and unique semi-structured interviews that were subsequently submitted to Content Analysis. Results: There was a disparity in the technical-scientific and practical knowledge of nursing professionals. There were weaknesses in carrying out guidelines and in the preoperative preparation involved in the ostomy process, in addition to the lack of knowledge about the physiological processes that change with surgery. However, there were professionals who stood out for their technical-scientific domain and for their differentiated care for people with intestinal ostomy, seeking to meet their specificities and providing guidance based on the level of understanding of the person under their care and their family. Final Considerations: There is a weakness in the care provided to the person with an ostomy and their family, however, continuing education together with the implementation of the Nursing Care Systematization in Surgical Clinic Units, are capable of generating knowledge from the reality of the patients. professionals and help them to build specialized care, resulting in the reduction of immediate or late complications, improving the experience of the transition of the person with intestinal ostomy to self-care.

KEYWORDS: Ostomy; Nursing; Nursing care; Self-care; Perioperative nursing. 


\section{INTRODUÇÃO}

Dados do Instituto Nacional do Câncer mostram que neoplasia de cólon e reto são a segunda causa mais comum de câncer ${ }^{1}$. Esse grande número de diagnósticos, associado à outras comorbidades intestinais, leva ao aumento do número de cirurgias para confecções de estomias intestinais, causando alterações físicas e psicológicas. Entretanto, comumente se observa a práxis da enfermagem direcionada à dimensão fisiológica, com abordagens psicossociais deficientes, sendo essa uma barreira na assistência com repercussões na vida da pessoa com estomia ${ }^{2,3}$.

A atenção à pessoa com estomia deve ser realizada por uma equipe especializada e multidisciplinar, promovendo a reabilitação enfatizada no autocuidado e na prevenção de complicações da estomia ${ }^{4}$. A partir da implementação da Sistematização daAssistência de Enfermagem (SAE), o enfermeiro é capaz de garantir cuidado humanizado, individualizado e integral, atendendo assim as necessidades de saúde da pessoa com estomia intestinal que transcendem as ações do processo de saúde-doença e estendem-se a um cuidado integral e holístico o qual visa contemplar as demandas externas e ambientais do indivíduo, a fim de proporcionar um vínculo entre o profissional e o paciente, interferir positivamente no seu bem-estar e estimular a prática do autocuidado ${ }^{5,6}$.

Frente às necessidades de saúde da população estomizada, os serviços de saúde precisam garantir educação permanente e continuada dos profissionais, além de adequadas estruturas físicas e recursos para efetivar planos de cuidados e protocolos de intervenção assistencial especializada a fim de oferecer assistência de qualidade ${ }^{7}$. Na unidade de clínica cirúrgica frequentemente as pessoas com estomias são internadas para a realização de procedimentos cirúrgicos, diagnósticos, ou para investigação de fatores associados à prática cirúrgica, momento em que atuação de enfermagem mostra-se essencial.

Neste sentido, a questão que norteia este estudo é: Como os profissionais de enfermagem da Unidade de Clínica Cirúrgica desenvolvem o cuidado à pessoa com estomia intestinal? Assim, objetivou-se conhecer o processo de cuidado à pessoa com estomia intestinal realizado pelos profissionais de enfermagem da Unidade de Clínica Cirúrgica.

\section{MÉTODO}

Pesquisa qualitativa, exploratória e descritiva desenvolvida na Unidade de Clínica Cirúrgica de um Hospital Universitário no extremo Sul do Brasil. A unidade possui 32 leitos destinados à situações cirúrgicas, tendo uma equipe de enfermagem formada por 35 profissionais, entre enfermeiros, técnicos, auxiliares, distribuídos em quatro turnos.

Participaram do estudo os profissionais da equipe de enfermagem que atenderam aos critérios de inclusão: atuar no setor há, pelo menos, seis meses e possuir experiência com o cuidado de pessoas com estomia intestinal e suas famílias. Excluiu-se os profissionais em férias, folguistas ou em licença saúde no período da coleta de dados. Todos os 
profissionais foram convidados sendo que oito não preencheram os critérios de inclusão e exclusão e sete se negaram a participar da pesquisa. Assim, participaram do estudo 20 profissionais da equipe de enfermagem.

A coleta de dados se deu nos meses de fevereiro a abril de 2019, por meio de entrevista única e individual, utilizando um instrumento semiestruturado contendo dados de identificação dos participantes e questões norteadoras abertas que envolviam o entendimento do entrevistado sobre o conceito de estomias, causas da necessidade da confecção de estomias intestinais, conhecimento sobre as possíveis complicações relacionadas ao estoma, bem como as suas causas, além da visão dos profissionais em sobre as consequências ocasionadas pelo processo de estomização na vida da pessoa que a possui.

Foi questionado também aos profissionais da Unidade de Clínica Cirúrgica sobre como se deu o seu preparo técnico-científico acerca da temática, bem como a sua vivência ao realizar os cuidados à pessoa com estomia intestinal e como os realiza, e quais as dificuldades identificadas nesta assistência, além da rotina de orientações ao paciente no pré e pós- operatório e a inserção da família neste processo de cuidado. As mesmas foram gravadas na integra e posteriormente transcritas e submetidas à Análise de Conteúdo de Bardin

Respeitou-se a Resolução 466/129 que rege as pesquisas envolvendo seres humanos, o projeto de CAAE $n^{0}$ 03771818.1.0000.5324 obteve parecer favorável, sob n² 282/2018 do Comitê de Ética em Pesquisa, cumprindo os princípios de sigilo e respeito ao participante em sua dignidade e autonomia mediante a assinatura do Termo de Consentimento Livre e Esclarecido. A identificação dos participantes foram feitas pela letra inicial da categoria sendo enfermeiros "E", técnicos "T" e auxiliares "A", seguidos do número da entrevista como forma de garantir seu anonimato. A distinção entre categorias foi utilizada com o intuito de analisar as etapas do processo de cuidado à pessoa com estomia intestinal, tendo em vista que cada categoria profissional apresenta diferentes atribuições.

\section{RESULTADO}

Entre os 20 participantes da pesquisa estavam oito auxiliares, oito técnicos e quatro enfermeiros, com idades que variaram de 31 anos a 63 anos, nove dos profissionais possuíam 50 anos ou mais. Quanto ao sexo, 17 eram do sexo feminino e apenas três do sexo masculino. 0 tempo de exercício da enfermagem variou de sete meses a 36 anos, porém 12 dos entrevistados tinham 20 anos ou mais de exercício profissional.

\section{As fragilidades nas orientações prestadas pela enfermagem}

As orientações são um ponto importante do cuidado de enfermagem para a reabilitação plena da pessoa e família. Entretanto, os relatos nos mostram a fragilidade na realização desse cuidado o qual não é feito pela falta de tempo. 
Não. Geralmente as orientações não são dadas, falando especificamente de ostomia. (T2)

Quando a gente tem tempo, quando estamos com poucos pacientes, até dá para dar uma atenção maior. Mas no geral é muito corrido. Não dá tempo para gente orientar, conversar direitinho. (A1)

Observa-se que não há uma sistematização sobre o momento de orientar sobre os cuidados com a estomia. Há profissionais que não orientam, outros o fazem durante a realização do cuidado ou quando há previsão de alta hospitalar.

Quando o paciente está tendo alta é orientado sobre os cuidados e troca da bolsa para tentar fazer que o paciente seja o mais independente, tentando ajudar na troca. Eu levo ele no banheiro para mostrar como vai ser quando ele for para casa, para não precisar pedir para as pessoas e ser independente. Tem um grupo que orienta também. (T1)

A orientação é sempre quando a gente vai trocar a bolsa, que a pessoa olha e a gente já ensina a trocar a bolsinha. Se o paciente está há muito tempo internado, ele mesmo já troca a bolsinha, já limpa e vai fazendo as rotinas que serão feitas em casa.

Verifica-se que não há uma sistematização das orientações ainda no pré-operatório além do preparo do intestino, até mesmo porque há pacientes submetidos à estomização de forma emergencial sendo orientado no pós-operatório, além disso, as orientações são vistas como uma função do médico e do enfermeiro.

Geralmente, a gente como técnico não faz nada. O médico e a enfermeira que vão e orientam o paciente sobre o procedimento. (T7)

É o preparo do intestino. Bisacodil e manitol com suco de laranja ou limão. (A2)

É de dar apoio ao paciente, porque não é fácil tu vir do bloco com uma bolsa de colostomia. (A5)

A gente faz os cuidados de orientar os pacientes, tentar preparar ele para o que vai acontecer. (E4)

Dificilmente tu sabe quando ele vai fazer uma colostomia, em princípio ele vai para o bloco para fazer uma correção de tumoração, obstrução, e os cuidados que a 
gente tem são baseados em orientações, pois é fundamental. Quando ele chega a gente faz orientações com os cuidados com o estoma. (E3)

Apesar das fragilidades observa-se que as orientações dadas possuem o foco em assuntos importantes de serem realizados ainda na internação como o cuidado com a bolsa coletora e a pele, os produtos de cuidado e as situações que podem ser vivenciadas pela pessoa agora estomizada.

Mostrar os produtos, explicar como fazer. Orientar a não trocar a bolsa com muita frequência que pode dar alergia. Explicar que não é um procedimento estéril e pode ser lavado com água normal. Eles têm que ali é estéril e eu explico que é como se fosse um ânus. E em toda troca à gente lava a bolsinha para não ficar odor. Explico sobre os ruídos e odores que podem sair. Tento fazer com que a pessoa não fique constrangida porque são barulhos involuntários e explicar para deixar a bolsinha sempre mais vazia para não pesar e descolar. (T1)

A higiene, explicar que não é a pior coisa do mundo, que ele vai ir se adaptando.

\section{Condutas no cuidado das pessoas com estomias intestinais}

Ao desenvolverem as rotinas assistenciais de cuidado os profissionais realizam diferentes condutas que devem ser baseados no conhecimento cientifico e que vão justificar o cuidado. Entretanto, observa-se que essas nem sempre se baseiam no conhecimento científico ou raciocínio clínico esperado do profissional preparado para tal cuidado.

É, esvaziar a bolsa e higienizar. Uso água destilada para lavar. A limpeza é feita sempre que necessário. A troca da bolsa é feita somente pela enfermeira. (A4)

A limpeza da bolsa é com soro fisiológico e esvaziar geralmente é conforme a necessidade. A troca da bolsa é quando necessário. Eu limpo a pele, seco e eu faço o recorte a olho. (A8)

A lavagem, o esvaziamento e cuidados com a queimadura. Eu faço a limpeza com água. Lavo bem lavado e desprezo. A troca quem faz é o enfermeiro. Mas numa urgência a gente faz. A gente troca quando descola. Lava, seca e coloca os produtos, mede o estoma e cola. (T3)

E também cuidar para fazer o recorte da bolsa certinho. Eu sempre uso as 
réguas que vem e tento lavar sempre direitinho a bolsa. Uso as luvas estéreis e sempre cuido para não contaminar. (E4)

Esse despreparo pode estar alicerçado na falta de preparo formal ou experiência no cuidado como competências técnicas e orientações. Entretanto, se observa que a experiência no cuidado fortalece o profissional para o exercício do cuidado.

A experiência aqui é bem o que vemos no curso. Aqui não recebemos uma orientação adequada do setor de estomizados. (T6)

Acho que foi normal, a gente fazia a limpeza e depois dava as orientações para quando ela tivesse alta. (T7)

No início foi complicado pois estava trabalhando num local onde eu era a única enfermeira e nunca tinha atendido ninguém, só tinha visto nos livros. (E4)

Acerca das rotinas de cuidado perioperatórios observa-se que há profissionais que realizam orientações voltadas para pessoas com estomias, mas também há quem não visualize as especificidades dessa população realizando apenas cuidados gerais o que revela a falta da sistematização da assistência de enfermagem fragilizando o cuidado.

Mesmos cuidados que tem com os outros pacientes cirúrgicos (...). Mas não tem nenhum cuidado assim específico. (A3)

Faz o cuidado da bolsa e orienta também o manuseio, procurar o grupo de estomizados, explica dos produtos específicos, orienta tomar banho. (T6)

A família possui papel importante na reabilitação da pessoa com estomia precisando ser inserida no processo de cuidado. Observa-se a falta de padronização, sendo a família ora orientada em um grupo de estomizados, ora pelo enfermeiro da Unidade de Clínica Cirúrgica, sendo essas reforçadas pelos técnicos e auxiliares de enfermagem quando o familiar demonstra interesse no cuidado.

Sim, normalmente a família é inserida no cuidado porque provavelmente ele vai para casa com estomia, sendo capaz da família realizar o cuidado ou até mesmo o próprio paciente. (E1)

Ali no balcão tem o papel que informa do grupo de estomia. Que os pacientes e familiares que querem aprender vão lá. Aqui os pacientes recebem orientação das 
enfermeiras e a gente quando vai fazer o cuidado também explica direitinho como lavar. Às vezes tem familiar interessado, que quer ver como faz, quer fazer o cuidado e nós ensinamos, mas quem ensina mesmo é o enfermeiro. Mas se um familiar chega em mim e pede para fazer e para auxiliar eu sempre ajudo e deixo ele fazer o processo. Mas a preferência é que elas vão no grupo dos estomizados. (A7)

Sim recebe orientações. Geralmente as orientações quem dão são as enfermeiras. A gente só faz o cuidado e reforça as orientações. Mas lidar com pessoas é sempre complicado. Tem o NPO que não respeitam. Tem coisas que não temos como lidar. As orientações são repassadas, mas os familiares complicam muito. (T8)

Muitos profissionais têm relacionado à experiência ao lidar com pessoas com estomia intestinal com as dificuldades encontradas ao realizar o cuidado, dentre as dificuldades citadas tem-se a falta de materiais, o número reduzido de funcionários, a confecção e tipo de estoma, a reação do paciente e familiares frente ao processo de estomização e a falta de tempo para um atendimento direcionado.

Às vezes tem pouco funcionário. (A1)

[...]o paciente que complica quando tem ileostomia[...] (A4)

É lidar com a falta de aceitação do paciente. (T3)

Lidar com os pacientes e familiares que não tem uma boa aceitação. Às vezes, a gente não tem material, tanto a bolsa quanto os produtos. (T6)

Em algumas a gente encontra dificuldades em função da maneira como é exteriorizada, ou a sutura que é feita em torno. (E3)

\section{DISCUSSÃO}

No exercício da enfermagem é comum a existência de práticas que exigem domínio prático, destreza e treino. Tornando os profissionais de enfermagem seres mecanizados, com raciocínio técnico em que o conhecimento empírico passa a ser dominante e automatizado do processo desvirtua o raciocínio clínico e pensamento crítico. Assim, acreditase que o desenvolvimento do processo de trabalho da enfermagem deve ir além da mecanização de procedimentos e contemplar bases sólidas de conhecimentos, prevenindo que a transmutação entre ciência da enfermagem e conhecimento 
empírico proporcione incertezas, vulnerabilidades da assistência e variações de práticas de cuidado ${ }^{10}$.

Frente às especificidades da pessoa estomizada, o parecer 04/2016 do COFEN, destina que o profissional indicado para o cuidado da pessoa com estomias é o enfermeiro estomaterapeuta, pois este é capaz de promover ações de planejamento e execução de um plano de cuidados específico para cada paciente e avaliar a sua implementação. No entanto, não há no mercado profissional uma proporção adequada de enfermeiros estomaterapeutas, sendo assim, frequentemente, o enfermeiro generalista é o responsável pelas ações de cuidado à pessoa com estomia ${ }^{11}$.

As ações pré e pós-operatórias precisam ser fortalecidas para a resolução da complexidade de se tornar uma pessoa com estomia. Pois, a falta de preparo para a vivência da estomização por parte da pessoa que se submeterá a essa intervenção e as ações insuficientes dos profissionais pode acarretar, comumente, em lesões periestomais que prejudicam a reinserção da pessoa com estomia em seu novo contexto em virtude do desconforto, dor e incertezas em decorrência da lesão peristomia ${ }^{12}$.

Compreende-se que a construção de saberes e conhecimentos dos profissionais de enfermagem proporciona benefícios à pessoa com estomia, pois este terá bases fundamentadas para orientação do processo de trabalho e assim garantir segurança e motivação a pessoa estomizada e sua família. É de grande valia que a enfermagem adquira conhecimentos sobre o planejamento e organização das ações do processo de cuidado e assim desenvolvam ações de capacitação para todos os membros da equipe, sendo estes capaz de manejar mais diversas situações de saúde ${ }^{13}$.

A disparidade entre teoria e prática no cuidado à pessoa estomizada compromete a qualidade de vida (QV) desta $^{14}$. O desconhecimento do profissional de enfermagem sobre as etapas do processo de cuidado à pessoa com estomia pode levar a uma interpretação inadequada pelo paciente sobre a qualidade da assistência e induzir que este questione sobre as capacidades e competência da equipe de enfermagem, acarretando em distanciamento da pessoa com estomia intestinal dos profissionais de saúde ${ }^{15}$.

Diante da dinâmica hospitalar, o enfermeiro frente às atividades gerenciais, assistenciais e burocráticas, deve buscar novos saberes, atualizar-se sobre metodologias de trabalho, constituir um planejamento assistencial e estruturar uma equipe sólida e capacitada. O enfermeiro deve proporcionar a troca de conhecimentos junto à equipe, elaborar momentos para discussão de situações e condutas, criar rotinas assistenciais e planos de cuidado, bem como buscar a elaboração e a implementação de protocolos operacionais padrão, objetivando garantir uma assistência de qualidade e com procedimentos padronizados e com menos defasagem no processo de cuidado ${ }^{13}$.

Conforme a Política Nacional de Educação Permanente em Saúde se busca a transformação da prática profissional, fundamentada no processo de trabalho com responsabilidade no cuidado. Uma estruturação de estratégias de formação para os profissionais do SUS, com processos educativos descentralizado, ascendente e transdisciplinar ${ }^{16}$. 
Reforça-se que a educação permanente atua como uma metodologia de ensino fundamentada nas atividades diárias executadas, podendo assim ser discutidas as situações reais, ampliando o pensamento crítico na tomada de decisões ${ }^{17}$.

A educação continuada é essencial no desenvolvimento do profissional de saúde, pois é capaz de estimular um pensamento crítico e raciocínio clínico diante do cotidiano de atuação, construção de conhecimentos e habilidades. Cabe assim, às instituições de saúde estimularem e proporcionarem meios para a realização de ações de ensino para os profissionais, adequando as temáticas de acordo com as demandas de cada unidade, proporcionando condições que colaborem com a adesão dos profissionais de saúde, além também de incentivar à participação destes em eventos e cursos $^{13}$.

Assim, observou-se que há fragilidades no processo de educação continuada dos profissionais entrevistados, visto que uma proporção destes referiram práticas de cuidados à pessoa com estomia intestinal que revelam lacunas em seus conhecimentos técnicos e científicos. Conforme o Código de ética dos Profissionais de enfermagem (2017) é dever do profissional aprimorar seus conhecimentos técnicos, científicos, éticos e culturais em benefício da pessoa, família ou população, bem como garantir a proteção destes contra danos ocorridos em função de imperícia, negligência ou imprudência por parte da equipe de saúde ${ }^{18}$.

Os cuidados de enfermagem à pessoa com estomia intestinal vão além dos cuidados práticos como a higienização e troca da bolsa coletora. Deve-se abranger, também, a rotina de orientações a esta pessoa e sua família, tanto no préoperatório como no pós-operatório. Deve-se reforçar a prática do autocuidado quando a condição clínica da pessoa permitir, estabelecendo a participação da família no cuidado, tendo em vista que inicialmente este é quem assume as rotinas de cuidado após alta hospitalar, e nos casos quando a pessoa com estomia é incapaz de realizar o autocuidado, este é responsável pelos mesmos ${ }^{12}$.

O enfermeiro é responsável por prestar instruções à pessoa com estomia intestinal e sua família, relacionadas aos cuidados no pós-operatório, técnicas de manuseio com a bolsa coletora, cuidados com a pele periestomia e uso de adjuvantes. Deve orientar sobre a importância de buscar o Serviço de Estomaterapia, que presta assistência especializada a esta pessoa e realiza distribuição dos materiais necessários para o cuidado. Deve demonstrar empatia e segurança e assegurar-se que as orientações vão de encontro às necessidades da pessoa a ser cuidada e seu nível de entendimento ${ }^{14,19}$.

A responsabilidade de orientações à pessoa com estomia intestinal e sua família foi atribuída por muitos membros deste estudo como uma competência do Serviço de Estomaterapia (SE). O SE é regulamentado pela Portaria $N^{\circ} 400$, de 16 de novembro de 2009, e presta uma assistência multidisciplinar à pessoa com estomia após sua alta hospitalar. Tem ênfase nas consultas de enfermagem, cadastro de pessoas estomizadas, distribuição de materiais e adjuvantes 
para o cuidado com a estomia e pele periestomia, promoção do autocuidado, atenção às necessidades psicossociais, emocionais e espirituais da pessoa com estomia. A Secretária Estadual de Saúde é o setor responsável pela aquisição dos materiais necessário para distribuição às pessoas com estomias cadastradas no programa ${ }^{20}$.

\section{CONSIDERAÇÕES FINAIS}

O presente estudo atingiu seu objetivo ao conhecer o processo de trabalho da equipe de enfermagem no cuidado de pessoas com estomias intestinais. A pesquisa apresenta a limitação de ter sido realizada em um único cenário não permitindo generalizações.

Observaram-se as fragilidades na assistência devido à falta da SAE observada pela falta de planejamento, padronização e metas no cuidado, cuidados errôneos devido à falta da educação permanente e continuada além da incompreensão dos papeis da equipe de enfermagem no cuidado da pessoa com estomia. Entretanto, se observa que os profissionais dedicam-se dentro de suas possibilidades de cuidado.

A partir dos resultados recomenda-se a implementação de ações de educação permanente e continuada com temáticas pertinentes ao cotidiano do cuidado das unidades buscando preparar a equipe para atender as especificidades de cada paciente. Além disso, a implementação da SAE favorece a assistência humanizada, individualizada e integral, que visa contemplar os aspectos biopsicossociais, emocionais e espirituais de cada pessoa, garantindo assim QV à pessoa com estomia intestinal.

\section{REFERÊNCIAS}

1. Instituto Nacional de Câncer. Ministério da Saúde. Estimativa de Câncer no Brasil,2020. MS / INCA / Coordenação de Prevenção e Vigilância / Divisão de Vigilância e Análise de Situação. Acesso em 25/05/2020. Disponível em: https://www.inca.gov.br/numeros-de-cancer

2. Corman ML, Bergamaschi RCM, Nicholls RJ, Fazio VW. Cirurgia Colorretal. $6^{\circ}$ ed. Thieme Revinter Publicações LTDA; 2017.

3. Cauduro FP, Schneider SMB, Menegon DB, Duarte ERM, Paz PO, Kaiser DE. Atuação dos enfermeiros no cuidado das lesões de pele. Recife, 2018. Rev. enferm UFPE on line. 2018; 12(10): 2628-34.

4. Brasil. Ministério da Saúde. Secretaria de Atenção à Saúde. Dispõe sobre as Diretrizes Nacionais para a 
Atenção à Saúde das Pessoas Ostomizadas no âmbito do Sistema Único de Saúde. Portaria n. 400, 16 novembro 2009. Diário Oficial da União, 2009.

5. Benedet SA, Gelbcke FL, Amante LN, Padilha MIS, Pires DP. Processo de enfermagem: instrumento da sistematização da assistência de enfermagem na percepção dos enfermeiros. Care Online, 2016; 8(3):4780-4788.

6. Bezerra Sobrinho A, Vasconcelos AK A, Leite-salgueiro CDB. O Cuidado Integral como uma Missão da Enfermagem: Uma Revisão Integrativa da Literatura. Id on Line Rev.Mult. Psic., 2018; 12(42): 790-804. ISSN: 1981-1179.

7. Sasaki VDM, Teles AAS, Lima MS, Barbosa JCC, Lisboa BB, Sonobe HM. Reabilitação de pessoas com estomia intestinal: revisão integrativa. Recife, 2017. Rev. enferm UFPE on line. 2017; 11(4): 1745-54.

8. Bardin L. Análise de conteúdo. Ed. 70 São Paulo: Persona; 2011.

9. Brasil. Ministério da Saúde. Conselho Nacional de Saúde. Resolução n. 466, de 12 de dezembro de 2012. Aprova diretrizes e normas regulamentadoras de pesquisas envolvendo seres humanos. Brasília. Diário Oficial da União, 2012.

10. Queiróz PJ. Enfermería, una ecología de los saberes. Portugal, 2016. Cultura de los Cuidados (Edición digital). 2016; 20(45): 137-146.

11. Conselho Federal de Enfermagem (COFEN). Resolução COFEN nº 04, de 2016. Dispõe sobre a Manifestação sobre procedimentos da área de enfermagem. Brasília. COFEN, 2016.

12. Nichols T. Health Utility, Social Interactivity, and Peristomal. EUA, 2018. Skin Status Journol Wound Ostomy Continence Nurs. 2018; 45 (5): 438-443.

13. Soares MI, Camelo SHH, Resck ZMR, Terra FS. Nurses' managerial knowledge in the hospital setting. Rev Bras Enferm [Internet]. 2016; 69(4): 631-7. 
14. Moraes T, Santos CF, Borges EL. Da formação à prática: a percepção de supervisores de enfermagem sobre os cuidados em estomias. Rio de Janeiro, 2016. Rev enferm UERJ. 2016; 24(2): 1-6.

15. Oliveira LN, Lopes APA, Decesaro MN. Cuidado integral à pessoa estomizada na atenção básica - conhecimento e atuação do enfermeiro. Maringá, 2017. Ciencia Cuidado e Saude. 2017; 16(3): 1-8.

16. Brasil. Ministério da Saúde (MS). Política Nacional de Educação Permanente e Desenvolvimento para o SUS - Caminhos para Educação Permanente em Saúde. Brasília. MS, 2004.

17. Carvalho NR, Toledo LV, e Silva EA, Figueiredo JB, Estevão ASC, de Oliveira DM. A construção de saberes através da educação permanente na atenção básica à saúde: relato de experiência. J Manag Prim Health Care [Internet]. 2017; 7(1): 122.

18. Conselho Federal de Enfermagem (COFEN). Resolução COFEN Nº 564/2017. Novo Código dos Profissionais de Enfermagem. Brasília. COFEN: 2017.

19. Mazon LM, Piccini E. A realidade e os desafios do enfermeiro na assistência à pessoa ostomizada. Brasil, 2015. Saúde Meio Ambiente. 2015; 4(1): 117-28.

20. Brasil. Ministério da Saúde. Secretaria de Atenção à Saúde. Dispõe sobre as Diretrizes Nacionais para a Atenção à Saúde das Pessoas Ostomizadas no âmbito do Sistema Único de Saúde. Portaria n. 400, 16 novembro 2009. Diário Oficial da União, 2009. 